PHYSICAL REVIEW D 89, 123004 (2014)

\title{
Correlating Fourier phase information with real-space higher order statistics in CMB data
}

\author{
H. I. Modest, ${ }^{1,{ }^{*}}$ C. Räth, ${ }^{1}$ A. J. Banday, ${ }^{2,3}$ K. M. Górski, ${ }^{4,5}$ and G. E. Morfill ${ }^{1}$ \\ ${ }^{1}$ Max-Planck Institut für extraterrestrische Physik, PO Box 1312, Giessenbachstraße 1, \\ D-85748 Garching, Germany \\ ${ }^{2}$ Université de Toulouse, UPS-OMP, IRAP, F-31028 Toulouse CEDEX 4, France \\ ${ }^{3}$ CNRS, IRAP, 9 Av. Colonel Roche, BP 44346, F-31028 Toulouse CEDEX 4, France \\ ${ }^{4}$ Jet Propulsion Laboratory, California Institute of Technology, Pasadena, California 91109, USA \\ ${ }^{5}$ Warsaw University Observatory, Aleje Ujazdowskie 4, 00 - 478 Warszawa, Poland
}

(Received 11 October 2013; revised manuscript received 24 January 2014; published 5 June 2014)

\begin{abstract}
We present a heuristic study on the correlations between harmonic space phase information and higherorder statistics. Using the spherical full-sky maps of the cosmic microwave background as an example, we demonstrate that known phase correlations at large spatial scales can gradually be diminished when subtracting a suitable best-fit (Bianchi-)template map of a given strength. The weaker phase correlations are attended by a vanishing signature of anisotropy when measuring the Minkowski functionals and scaling indices in real space with the aid of surrogate maps being free of phase correlations. Those investigations can open a new road to a better understanding of signatures of non-Gaussianities in complex spatial structures, especially by elucidating the meaning of Fourier phase correlations and their influence on higher-order statistics.
\end{abstract}

DOI: 10.1103/PhysRevD.89.123004

PACS numbers: 98.70.Vc, 07.05.Pj, 95.75.Pq, 98.80.Es

\section{INTRODUCTION}

Great advances in imaging techniques nowadays allow for a visualization of spatial structures in medicine, biochemistry, solid-state physics, or astronomy, ranging from the atomic nanometer scale [1] to megaparsec for the large scale structure of the Universe [2] with the largest and oldest observable structure being the cosmic microwave background (CMB) [3-5]. These images of complex natural structures contain a wealth of information about the origin and often nonlinear formation process of structure. As known from the field of signal processing and imaging science, for example, in optics, cybernetics, or time-series analysis, a comprehensive analysis of signals stemming from systems with nonlinear dynamics must go beyond a linear analysis (autocorrelation function in real space or the power spectrum in Fourier/harmonic space). In image analysis by higher-order statistics (HOS), this is only achieved when the phase information is included. A detailed understanding of this phase information has become very important in natural sciences in recent years and can improve existing methods of image analysis, image reconstruction, and also image compression [6]. Examples for studies on phase information and their application can be found in the development of the first phase retrieval methods in x-ray imaging [7-9] or in studies of the phase distribution in $\mathrm{CMB}$ data [10-12], inter alia.

* Corresponding author. hmodest@mpe.mpg.de
The higher-order $n$-point correlation functions with $n>2$ in real space or their equivalent polyspectra in Fourier space, however, do not allow direct conclusions on the distribution of the phases yet. If the signal is Gaussian, the Fourier phases are independent and identically uniform distributed. In this case, the second-order measures are anyway sufficient to understand the underlying physics. In generic cases, though, in which the underlying random fields are non-Gaussian, the phases are correlated and contain information that must not be neglected. One of the next steps on the way to a more profound understanding of images in general is a detailed description of the phase distribution and the investigation of the relation between phase information and real-space HOS.

In cosmology, the search for primordial non-Gaussian random fields has attracted great attention since their detection and identification allows for a differentiation between various models of inflation. While, e.g., multifield inflation or self-interactions of the inflaton field generally yield measurable non-Gaussianity (NG), the standard isotropic cosmology with the simple single-field slowroll inflationary scenario and a Friedmann-RobertsonWalker (FRW) metric predicts a Gaussian distribution for the first density perturbations of the Universe [13-15]. The latest, most precise measurements of parametrized NGs of the local, equilateral, and orthogonal types by the Planck team did not reveal significant deviations from Gaussianity [16]. A model-independent test using the well-established method of surrogates [17] applied to the Planck CMB maps revealed, however, NGs and hemispherical asymmetries for higher-order statistics [18], which can be traced back to 
harmonic space phase correlations on large spatial scales at low spherical harmonic modes $\ell$ with $\ell<20$, confirming previous findings in Wilkinson Microwave Anisotropic Probe (WMAP) data [19-21].

Evidence was found that a best-fit Bianchi type $\mathrm{VII}_{\mathrm{h}}$ template (BT) correlates with the large-scale anomalies in the CMB sky [22-24], although it is clear the best-fit Bianchi model itself is not compatible with the parameters of the cosmological concordance model (see, e.g., Ref. [25]). Bianchi models provide a generic description of anisotropic homogeneous cosmologies [26] that are only asymptotically close to a FRW universe. Applying a BT correction to CMB data yields a sky that is statistically isotropic for several subsets of statistical measures, e.g., the local power estimates [22]. In Ref. [18], it was found that the signal stemming from low $-\ell$ phase correlations can also be significantly reduced if the best-fitting BT is subtracted from the Planck maps. These results could hint at the properties of fully compliant cosmological models, especially when the behavior of the data is studied as a function of the BT correction and on isolated scales.

This work aims at the systematic investigation of the fundamental relation between the Fourier phase distribution in harmonic space and real-space higher-order statistics, comparable to the Wiener-Khinchin theorem [27,28]. To do so, we analyze the distributions of Fourier phases directly using the nonparametric statistical Kuiper test and compare these results to real-space signatures from higher-order correlations involving surrogates maps. The investigations are carried out using the $\mathrm{CMB}$ as an example of a spherical data set where no boundary conditions have to be met. We find that our previous real-space results studying the phase correlations of CMB data on the large scales with $\ell$ modes of $\ell<20$ are reproduced in a study with $\ell<10$. With this even stricter choice of $\ell$ interval, we make sure that our analysis of the phase distribution is comparable to the phase analysis in Ref. [12], where they have used exactly the same range. To modify the strength of phase correlations contained in the data, we make use of anisotropic Bianchi type $\mathrm{VII}_{\mathrm{h}}$ best-fit templates. We compare full-sky CMB maps of the WMAP experiment [29] and the Planck mission [5].

\section{METHODS}

Assuming that an image $I(x, y)$ can be represented in terms of linear superposition of (not necessarily orthogonal) basis functions $\beta_{i}(x, y)$ by $I(x, y)=\sum_{i} a_{i} \beta_{i}(x, y)$, the CMB map with its temperature anisotropies $\Delta T / T(\theta, \phi)$ at angular position $(\theta, \phi)$ can be expanded in orthonormal spherical harmonics $Y_{\ell m}$ as

$$
\Delta T / T(\theta, \phi)=\sum_{l=0}^{\infty} \sum_{m=-l}^{l} a_{\ell m} Y_{\ell m}(\theta, \phi),
$$

with the complex spherical harmonic coefficients

$$
a_{\ell m}=\int \mathrm{d} \mathbf{n} T(\mathbf{n}) Y_{\ell m}^{*}(\mathbf{n}),
$$

where $\mathbf{n}$ is the unit direction vector, $T$ is the CMB temperature anisotropy, $Y_{\ell m}^{*}$ are the complex conjugates of the spherical harmonics, and $a_{\ell m}=\left|a_{\ell m}\right| e^{i \varphi_{\ell m}}$. The phases $\varphi_{\ell m}$ of the harmonic coefficients are not rotational invariant. The set of the spherical harmonics is defined with respect to a particular coordinate system. For the CMB, the usual system is in Galactic coordinates.

Gaussianity of the CMB implies a Gaussian distribution of its independent complex spherical harmonic coefficients $a_{\ell m}$. According to theory, the harmonic space phases are then independent and identically distributed (i.i.d.) and follow a uniform distribution in the interval $[-\pi, \pi]$. We test for this null hypothesis of uncorrelated phases with two complementary methods to enable a comparison between them. Method A directly explores the distribution of the phases in harmonic space and is motivated by the findings in Ref. [12]. Method B is based on a real-space analysis and is supported by the method of surrogates. Generating the surrogate maps, we destroy only a single characteristic of the original map, which is a possible correlation of the phases. We gradually diminish the strength of phase correlations by subtracting a Bianchi type $\mathrm{VII}_{\mathrm{h}}$ best-fit template (taken from Ref. [30] for WMAP and taken from Ref. [31,32] for Planck) multiplied by a strength factor of $f=0.1,0.3,0.5,0.7,0.9$, and 1.0 from the original map to enable a comparison of the response in methods $\mathrm{A}$ and $\mathrm{B}$ as a function of the correction. Tests reveal so far that remaining Galactic plane foreground residuals and inpainting techniques in Planck SMICA and SEVEM and WMAP-9 ILC do not influence the low- $\ell$ phase correlations, which corresponds to findings in Refs. [18-20]. We will therefore use full-sky maps in our analysis.

\section{A. Method A-Phase space}

For a precise analysis of the phase distributions of the maps, we calculate the $a_{\ell m}$ coefficients of Planck SMICA and SEVEM [33] and WMAP-9 ILC [34] full-sky maps and test their phases $\varphi_{\ell m}$ for independence. We obtain 54 phases $\varphi_{\ell m}$ with values between $-\pi$ and $\pi$ depending on the chosen coordinate system after an $a_{\ell m}$ decomposition for $\ell \in[2,10], m>0$. If these $\varphi_{\ell m}$ fulfill the random phase hypothesis, i.e., are i.i.d. and follow a uniform distribution, the phase difference taken between these phases should be uniformly distributed in $[0,2 \pi]$. To test this, we define subsets of differences with fixed separations $(\Delta \ell, \Delta m)$ by $\Delta \varphi(\Delta \ell, \Delta m)=\varphi_{\ell+\Delta \ell, m+\Delta m}-\varphi_{\ell m}$. The Kuiper statistic $[35,36]$ is then used to test for the null hypothesis by comparing the cumulative distribution function (CDF) of the $\Delta \varphi(\Delta \ell, \Delta m)$ with a given uniform CDF. The Kuiper test statistic is $V=D^{+}+D^{-}$, where $D^{+}$and $D^{-}$represent 
the absolute sizes of the most positive and most negative difference between the two cumulative distribution functions that are being compared. For $N_{e}>4$, the $p$ value of an observed value $V$ is given by

$$
p=Q_{\mathrm{KP}}\left(\left[\sqrt{N_{e}}+0.155+0.24 \sqrt{N_{e}}\right] V\right),
$$

with respect to the monotonic function

$$
Q_{\mathrm{KP}}(\lambda)=2 \sum_{j=1}^{\infty}\left(4 j^{2} \lambda^{2}-1\right) e^{-2 j^{2} \lambda^{2}}
$$

where $N_{e}$ is the effective number of data points. The $p$ value can be interpreted as the probability of measuring a value at least equal to the observed value $V$ or even more extreme when the test statistic is applied to data that satisfies the null hypothesis. The smaller the $p$ value is, the more convincing the evidence is against the null hypothesis. High $p$ values for a given $(\Delta \ell, \Delta m)$ separation therefore indicate the absence of phase correlations between mode pairs separated by $(\Delta \ell, \Delta m)$, whereas low values indicate their existence. Values of $p \leq 0.05$ (5\% level) are widely accepted as strong evidence against the null hypothesis.

Our results depend on the chosen coordinate system. However, averaging over rotated systems will show a global trend of the results.

\section{B. Method B-Real space}

Using a shuffling approach, we generate surrogate maps by randomizing the potentially correlated phases $\varphi_{\ell m}$ of the original map while preserving the full-sky power spectrum of the map. In a prestep, we apply an initial Gaussian remapping of the temperature field and a uniform remapping of the phases to avoid any influence of data outliers on the measurement of phase correlations. If the original phases are independent, the shuffling process will statistically not influence the real-space higher-order statistics of the maps. Phase correlations that are contained in the original data are destroyed by the shuffling process. To enable a scale-dependent analysis of the maps, we generate one first-order surrogate and 200 second-order surrogate maps. In the first-order surrogate, only the phases with $\ell$ outside the $\ell$ range of $[2,10]$ are randomized. In a second step, we shuffle the remaining phases inside that $\ell$ range. Significant deviations between these two classes of surrogates reveal phase correlations in the original data among $\varphi_{\ell m}$ with $\ell \in[2,10]$.

To quantify the higher-order content of the surrogate maps, we use two comparable real-space image analysis methods sensitive to HOS, namely, the scaling index method, which calculates the weighted scaling indices $\alpha$ of the three-dimensional point distribution $P=\left\{\mathbf{p}_{i}\right\}$ of the CMB expressed by

$$
\alpha\left(\mathbf{p}_{i}, r\right)=\frac{\sum_{j=1}^{N_{\mathrm{pix}}} q\left(\frac{d_{i j}}{r}\right)^{q} e^{-\left(\frac{d_{i j}}{r}\right)^{q}}}{\sum_{j=1}^{N_{\mathrm{pix}}} e^{-\left(\frac{d_{i j}}{r}\right)^{q}}},
$$

as developed in Refs. [37,38], and a set of three statistics known as the Minkowski functionals [39],

$$
\begin{aligned}
M_{0} & =\int_{Q} \mathrm{~d} a \\
M_{1} & =\frac{1}{4} \int_{\partial Q} \mathrm{~d} \ell \\
M_{2} & =\frac{1}{2 \pi} \int_{\partial Q} \mathrm{~d} \ell k_{g},
\end{aligned}
$$

which were introduced into cosmology by Refs. [40-42]. For details and former results, see Ref. [21].

Calculating the Minkowski functionals, the surrogate temperature maps are divided into active and a nonactive parts by running over 200 threshold steps $\nu_{i}$ with $-4 \sigma_{T} \leq \nu_{i} \leq+4 \sigma_{T}$. Temperature values of the surrogate maps are counted as active if they lie above the temperature threshold step, whereas the other values are taken as nonactive. We then calculate area, perimeter, and Euler characteristic of the active pixels. These three Minkowski functionals are calculated for 768 overlapping hemispheres in the sky. To quantify the degree of agreement between the surrogates of the two different orders with respect to higher-order correlations found with the three Minkowski functionals $M_{0}$ (area), $M_{1}$ (perimeter), and $M_{2}$ (Euler), we calculate the mean of each Minkowski functional $M_{\star \text {, surro2 }}$ for $N=200$ realizations of the second-order surrogate, per hemisphere $h$ and threshold bin $\nu$,

$$
A:=\left\langle M_{\star, \text { surro2 }}(\nu, h)\right\rangle=\frac{1}{N} \sum_{m=1}^{N} M_{\star, \text { surro2 }}(m, \nu, h),
$$

and the standard deviation

$$
\sigma_{M_{\star, \text { surro2 }}}(\nu, h)=\left(\frac{1}{N-1} \sum_{m=1}^{N}\left(M_{\star, \text { surro2 }}(m, \nu, h)-A\right)^{2}\right)^{1 / 2}
$$

for $m=1, \ldots, N$. We combine the mean and standard deviation in a diagonal $\chi^{2}$ statistic, per hemisphere $h$, for the surrogates of first order,

$$
\chi_{M_{\star, \text { surrol }}}^{2}(h)=\sum_{j=0}^{\nu}\left[\frac{M_{\star, \text { surro1 }}(j, h)-A}{\sigma_{M_{\star, \text { surro } 2}}(j, h)}\right]^{2},
$$

and for the second-order surrogate maps,

$$
\chi_{M_{\star, \text { surro2 }}}^{2}(h, m)=\sum_{j=0}^{\nu}\left[\frac{M_{\star, \text { surro2 }}(m, j, h)-A}{\sigma_{M_{\star, \text { surro } 2}}(j, h)}\right]^{2} .
$$

Plots of the $\chi^{2}$ statistic can be found in Fig. 3 .

Finally, the degree of agreement between the two types of surrogates is quantified by the $\sigma$-normalized deviation $S$, 


$$
S\left(\chi_{M_{\star}}^{2}(h)\right)=\frac{\chi_{M_{\star \text { surrol }}}^{2}(h)-\left\langle\chi_{M_{\star \text { surro2 }}}^{2}(h)\right\rangle}{\sigma_{\chi_{M_{\star, \text { surro2 }}}^{2}}(h)},
$$

for each of the 768 hemispheres with $\left\langle\chi_{M_{\star} \text { surro2 }}^{2}(h)\right\rangle$ and $\sigma_{\chi_{M_{\star, \text { surro }}^{2}}}(h)$ denoting the mean and the standard deviation of $\chi_{M_{\star \text {, surro }}}^{2}(h)$. We obtain the $\sigma$-normalized hemispherical deviations $S\left(\chi_{M_{0}}^{2}(h)\right), S\left(\chi_{M_{1}}^{2}(h)\right)$, and $S\left(\chi_{M_{2}}^{2}(h)\right)$ (called $S$ values) between the surrogates of first and second order for the area, perimeter, and Euler characteristics. As for the Minkowski functionals, we calculate the mean $\langle\alpha(r)\rangle$ and standard deviation $\sigma_{\alpha(r)}$ of the scaling indices $\alpha\left(\mathbf{p}_{i}, r\right)$ for the set of 768 hemispherical maps. The differences of the two classes of surrogates are again quantified by the $\sigma$-normalized deviation $S$,

$$
S(Y)=\frac{Y_{\text {surro1 }}-\left\langle Y_{\text {surro2 }}\right\rangle}{\sigma_{Y_{\text {surro2 }}}},
$$

where $Y$ represents a diagonal $\chi^{2}$ statistic

$$
\chi_{\langle\alpha(r)\rangle, \sigma_{\alpha(r)}}^{2}=\sum_{j=1}^{2}\left[\frac{B_{j}-\left\langle B_{j}\right\rangle}{\sigma_{B_{j}}}\right]^{2},
$$

as a combination of the mean and the standard deviation where $B_{1}(r)=\langle\alpha(r)\rangle, B_{2}(r)=\sigma_{\alpha(r)}$.

It can be shown that method B is rotational invariant. Regardless of the chosen coordinate system, the $S$-value pattern on the sphere is preserved.

\section{RESULTS}

Comparing the results from the Kuiper statistic of 36 $(\Delta \ell, \Delta m)$ combinations with $\Delta \ell=0-5$ and $\Delta m=0-5$ in different coordinate systems, we find the trend that gradually subtracting the corresponding BT from the original map leads to increasing Kuiper $p$ values with increasing Bianchi factor $f$ if $p<0.1$ in the original data. This behavior is a strong indication for vanishing phase correlations due to BT correction in certain phase separation subsets depending on the chosen coordinate system. Figure 1 shows the significance levels for 12 combinations of $\Delta \ell=0,1$ and $\Delta m=0,1,2,3,4,5$ for all steps of BT corrections with respect to the Galactic coordinate system. The majority of the $(\Delta \ell, \Delta m)$-mode pairs has $p$ values that lie well above 0.05 and even above 0.1 in the original map as well as after the BT corrections. These results have a relatively high probability of above $10 \%$ to arise under the null hypothesis of random phases.

In rare cases, we find higher $p$ values in the original map and values below 0.1 after the BT correction. In the Galactic coordinate system, e.g., this is the case at $(\Delta \ell, \Delta m)=(5,0)$ in the original ILC9 map, where the full BT correction leads to $p=0.064$. For SMICA, this behavior is found for the correction with $0.3 \times \mathrm{BT}$ and $0.5 \times \mathrm{BT}$, for SEVEM at correction steps $0.1,0.3,0.5$, and 0.7 . The mode pair $(\Delta \ell, \Delta m)=(1,2)$ is another exception: The subtraction of the BT is almost not increasing the $p$ values that remain below the 3\% level for SMICA and ILC9, and below 6\% for SEVEM, with respect to the Galactic coordinate system. In Table I, we list the $p$ values of the original maps and the fully BT corrected maps for all subsets with at least one value $<0.1$ either in the original

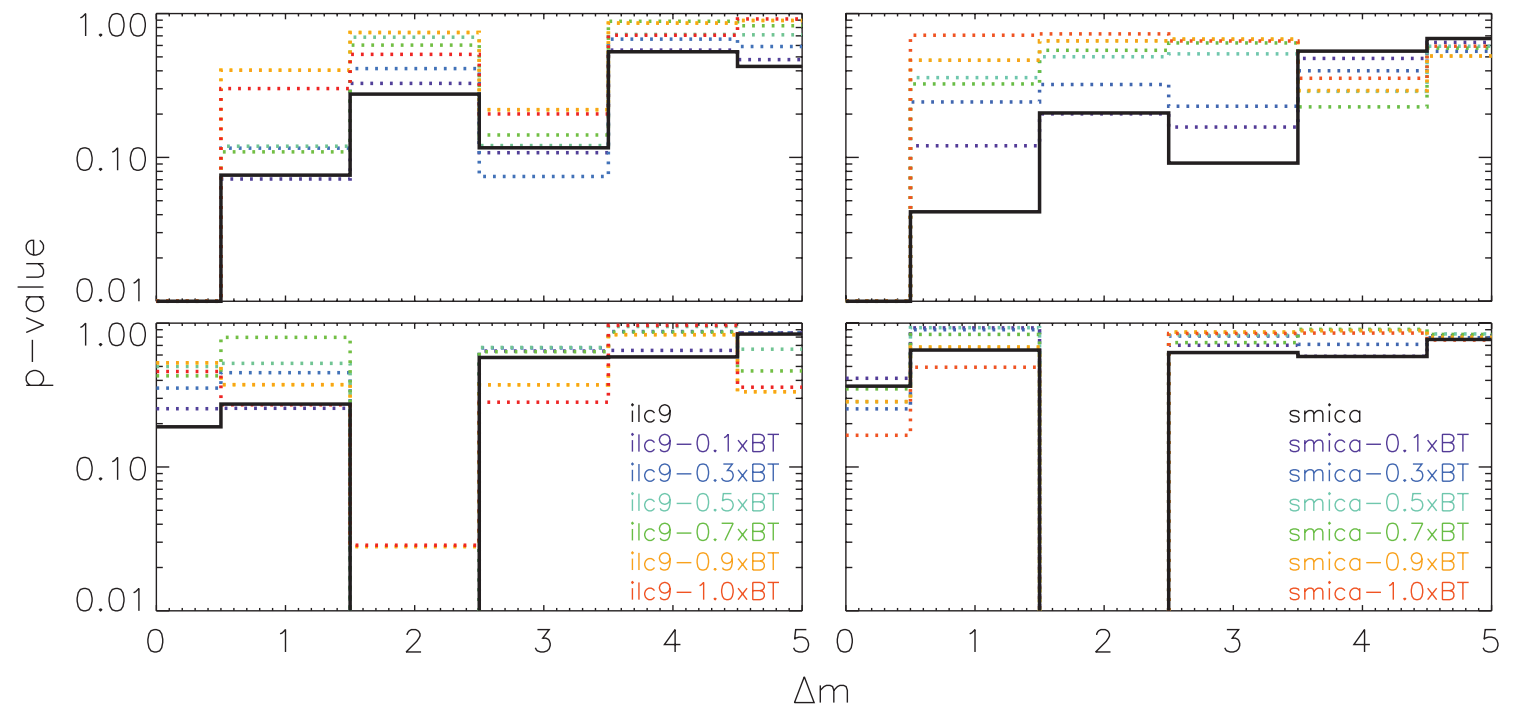

FIG. 1 (color). $\quad p$ values of the Kuiper statistic for the WMAP-9 ILC (left) and Planck SMICA map (right) (black solid line) and the corresponding Bianchi-corrected maps (colored dotted lines) for $\Delta \ell=0$ (top) and $\Delta \ell=1$ (bottom), calculated in the Galactic coordinate system. The Planck SEVEM map (not shown here) resembles the SMICA results. Note that subtracting the corresponding BTs does not significantly reduce the dip at $(\Delta \ell, \Delta m)=(1,2)$. 
TABLE I. Combinations of $(\Delta \ell, \Delta m)$ with $p$ values $<0.1$ (bold) for at least one of the three maps SMICA, SEVEM, and ILC9. We show $p$ before and after the full BT correction.

\begin{tabular}{|c|c|c|c|c|c|c|}
\hline \multirow[b]{2}{*}{$(\Delta \ell, \Delta m)$} & \multicolumn{2}{|c|}{ ILC9 } & \multicolumn{2}{|c|}{ SMICA } & \multicolumn{2}{|c|}{ SEVEM } \\
\hline & Original & Corrected & Original & Corrected & Original & Corrected \\
\hline$(0,1)$ & 0.075 & 0.301 & 0.042 & 0.707 & 0.160 & 0.853 \\
\hline$(0,2)$ & 0.275 & 0.520 & 0.204 & 0.722 & 0.096 & 0.916 \\
\hline$(0,3)$ & 0.117 & 0.201 & 0.091 & 0.645 & 0.242 & 0.526 \\
\hline$(1,2)$ & 0.003 & 0.029 & 0.002 & 0.005 & 0.008 & 0.060 \\
\hline$(2,5)$ & 0.090 & 0.193 & 0.087 & 0.471 & 0.151 & 0.454 \\
\hline$(5,0)$ & 0.321 & 0.064 & 0.148 & 0.126 & 0.132 & 0.196 \\
\hline$(5,4)$ & 0.364 & 0.218 & 0.220 & 0.328 & 0.078 & 0.139 \\
\hline
\end{tabular}

map or after the full BT correction with $1.0 \times \mathrm{BT}$. The findings in the original maps are consistent with those for the WMAP 3-year data tested by Ref. [12] and reveal an overall consistent picture of the different maps and experiments.

Averaging over 768 rotated coordinate systems reveals phase correlations for $\Delta \ell=0,1$ and $\Delta m=0,1,2$ but none for larger phase separations; see the Supplemental Material [43].

In Fig. 2, we show the inverse $|p-1|$ values with respect to the Bianchi factor $f$ for $\Delta \ell=0,1$ to visualize the trend in individual subsets (for the Galactic system). The mode pairs $(0,1),(0,2),(0,3)$, and $(2,5)$ (not shown) show a mostly monotonic decrease in the $p$ value with increasing $a$. The analysis of $(5,0)$ and $(5,4)$ (not shown) yields rather constant $p$ values above 0.75 . Mode pairs with $(1,2)$ remain correlated in all maps.

The $\chi^{2}$ statistic of the Minkowski functionals for 768 different hemispheres of the sky shows strong deviations between the first and 200 second-order surrogates generated from the original maps as can be seen in Fig. 3. The $\chi^{2}$ distribution of 768 first-order surrogate maps, generated from the Planck SMICA map, is after a BT correction very similar to the distribution of the second-order surrogates, which indicates that the originally contained phase correlations of the $\mathrm{CMB}$ are strongly reduced due to the subtraction of a Bianchi template. Analyzing the $\chi^{2}$ statistics of the Minkowski functionals and scaling indices by a comparison of surrogates 1 and 2 with respect to the local hemispherical contributions, method $\mathrm{B}$ reveals that both image analysis techniques detect similar asymmetries and deviations from Gaussianity in the CMB sky. This is true for the latest release of the SMICA and SEVEM map of Planck as well as for the WMAP-9 ILC data and does not depend on the chosen coordinate system. In Fig. 4, we show the color-coded $S$ values from a Minkowski Euler analysis for the 768 hemispheres, where a red pixel indicates strong deviations from Gaussianity in the hemisphere surrounding that pixel, and blue shows none. Subtracting the corresponding Bianchi type $\mathrm{VII}_{\mathrm{h}}$ best-fit templates from the Planck SMICA map diminishes phase correlations and gives an increasing isotropic Gaussian sky, which can be confirmed by an analysis with the scaling indices in Fig. 5. For the used SMICA map, the minimum

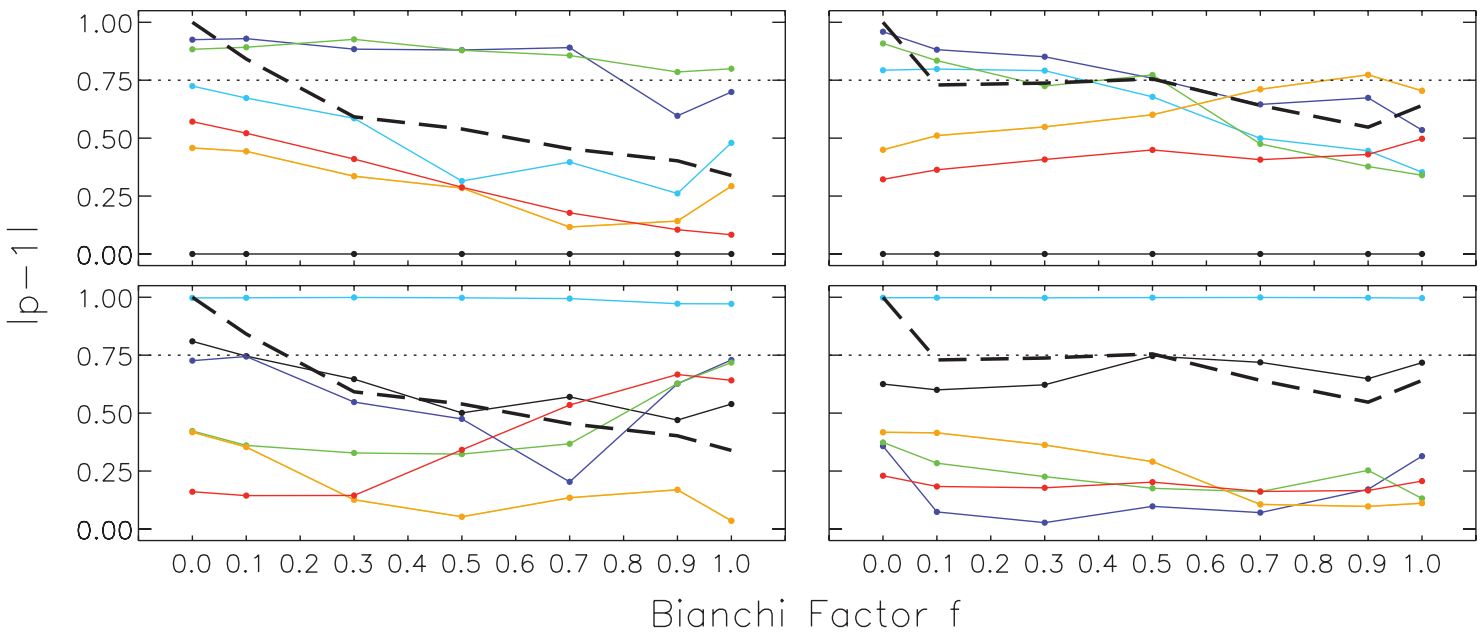

FIG. 2 (color). $|p-1|$ values of the ILC9 (left) and SMICA (right) map for $\Delta \ell=0$ (top) and $\Delta \ell=1$ (bottom). The colored lines mark the different $\Delta m$ with black $=0$, purple $=1$, blue $=2$, green $=3$, orange $=4$, and red $=5$. The black dashed line shows the normalized mean $S$ value of the output maps from method B (Fig. 4). 

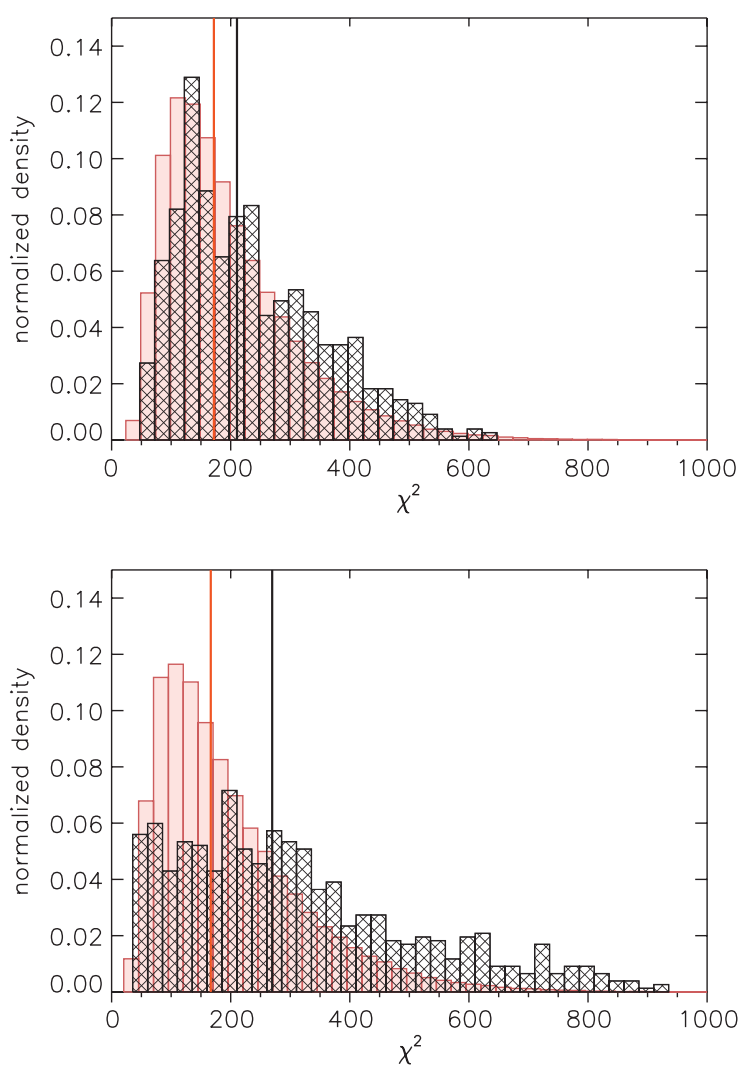

FIG. 3 (color). $\quad \chi^{2}$ distribution of the surrogates of first (black) and second (red) order generated from the Planck SMICA map, before (top) and after (bottom) the BT correction. The black and red lines indicate the corresponding median values of the histograms.

signal of deviations is particularly detected at $a=0.9$, not at 1.0. This is interesting in itself and requires further interpretation with respect to Bianchi template fitting and here especially to its $\ell$-range dependency.

To quantify the overall strength of deviation from the random phase hypothesis in method $\mathrm{B}$, we calculate the

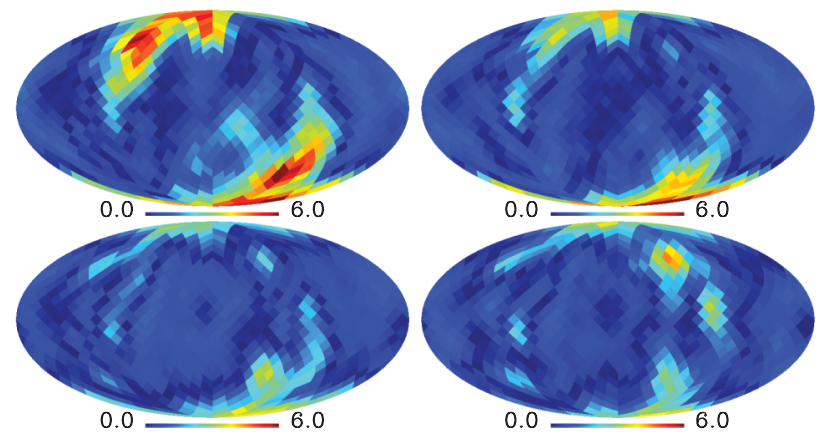

FIG. 4 (color). Mollweide projection in Galactic coordinates of the $S$-value distribution of method B using the Minkowski Euler characteristic for the Planck SMICA map. The BT is subtracted with a factor of $f=0.0,0.3,0.7,1.0$ (upper left to lower right), respectively.

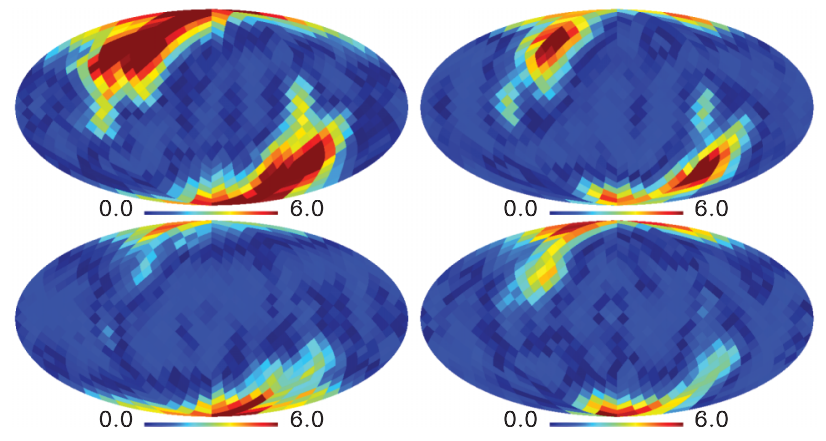

FIG. 5 (color). Same as Fig. 4 but for the the scaling index method.

mean of all $S$ values from the Minkowski analysis and show the result in Fig. 2. The comparison of method A and B reveals that the normalized mean of the real-space method B decreases with $a$, and likewise to the decrease as seen for mode pairs, e.g., $(\Delta \ell, \Delta m)=(0,1),(0,2),(0,3),(2,5)$ (for the SMICA map, in the Galactic system). Although method $\mathrm{B}$ reveals that the strength of the phase correlations is highly reduced after subtracting $1.0 \times \mathrm{BT}$, the $p$ value of $(\Delta \ell, \Delta m)=(1,2)$ remains almost constant.

\section{CONCLUSIONS AND OUTLOOK}

In a comparative study of the important and so far not understood relation between real-space higher-order statistics and the Fourier space phase information, we provide for the first time heuristic results using the example of the spherical CMB data. Analyzing the phase distribution of CMB maps on low $\ell$ modes, we detect a clear trend, but with low statistical significance, for gradually diminished phase correlations due to the subtraction of a Bianchi type $\mathrm{VII}_{\mathrm{h}}$ anisotropic cosmological template. This is especially true when looking at the subsets of "close-by" phase differences with $\Delta \ell=0-1$ and $\Delta m=0-3$ in the Galactic coordinate system. In comparison, we confirm a significantly vanishing higher-order signal of hemispherical asymmetries in the CMB sky for Bianchi-corrected maps. We suggest that the detected signatures of nonGaussianities and hemispherical asymmetries in real space due to phase correlations in the CMB can partly be explained by correlations between phases $\phi_{\ell m}$ separated by small $\Delta \ell$ and $\Delta m$.

In some subsets, the phase correlations are not diminished when subtracting the Bianchi template. Furthermore, the BT correction can even induce phase correlations for individual mode pairs to a certain extent, depending on the chosen coordinate system of the map. However, the statistic of the Kuiper test with such small effective numbers as used in this work is not strongly significant. The Bianchi template is not fully compatible with standard cosmological parameters, that fit very well to observations, and we cannot expect a perfect reduction of all existing anomalies, 
whether in real space or phase space. It is not solved yet whether individual phases $\phi_{\ell m}$ are responsible for the signatures of phase correlations or whether the relation between certain subsets plays a dominant role. On the studied very low $\ell$ range, instrumental noise is not an issue, and our method is neither influenced by residual foregrounds nor experimental systematics as shown in our earlier works. We therefore expect a cosmological explanation for the detected anomalies.

Low- $\ell$ anomalies are also detected in the linear regime of the data as, e.g., the power asymmetry, e.g., Ref. [44]; parity asymmetry, e.g., Ref. [45]; multipole alignment, e.g., Ref. [46]; or the lack of large-angle correlations, e.g., Ref. [47]. For an overview of the latest Planck results see Ref. [18]. It might point to a common physical origin of low- $\ell$ anomalies that they manifest themselves in the linear as well as nonlinear regime of the data.

Our results can contribute to an understanding of nonGaussian signals, which in cosmology may originate from early Universe physics but might also indicate a misinterpretation of cosmological events affecting the CMB. The existence of anomalies detected in the WMAP and Planck maps at large angular scales may even point to new fundamental physics. A detailed scale-dependent analysis of latest CMB data and a study of the coordinate system dependencies with respect to anisotropic cosmologies can give further insight into the constraints of fully compliant cosmological models. In summary, the combined analysis of phase statistics including their variations due to either template subtraction or refined surrogate generating methods, and of the respective response of higher-order statistics, offers a new statistical framework to disentangle the information content of images.

\section{ACKNOWLEDGMENTS}

We greatly thank Theresa Jaffe and Jason McEwen for providing us with the latest WMAP and Planck Bianchi templates, respectively. For the calculations, we employ the HEALPix software [48]. H. I. M. acknowledges the support of the Christiane Nüsslein-Volhard (CNV) foundation and the International Max Planck Research School.
[1] G. Binnig, C. F. Quate, and C. Gerber, Phys. Rev. Lett. 56, 930 (1986).

[2] C. P. Ahn et al., Astrophys. J. 203, 21 (2012).

[3] G. F. Smoot, C. L. Bennett, A. Kogut, E. L. Wright, J. Aymon, N. W. Boggess, E. S. Cheng, G. de Amici, S. Gulkis, M. G. Hauser, G. Hinshaw, P. D. Jackson, M. Janssen, E. Kaita, T. Kelsall, P. Keegstra, C. Lineweaver, K. Loewenstein, P. Lubin, J. Mather, S. S. Meyer, S. H. Moseley, T. Murdock, L. Rokke, R. F. Silverberg, L. Tenorio, R. Weiss, and D. T. Wilkinson, Astrophys. J. Lett. 396, L1 (1992).

[4] C. L. Bennett, M. Halpern, G. Hinshaw, N. Jarosik, A. Kogut, M. Limon, S. S. Meyer, L. Page, D. N. Spergel, G. S. Tucker, E. Wollack, E. L. Wright, C. Barnes, M. R. Greason, R. S. Hill, E. Komatsu, M. R. Nolta, N. Odegard, H. V. Peiris, L. Verde, and J. L. Weiland, Astrophys. J. Suppl. Ser. 148, 1 (2003).

[5] P. A. R. Ade et al.. (Planck Collaboration), arXiv: 1303.5062 .

[6] J. M. Rodenburg, A. C. Hurst, A. G. Cullis, B. R. Dobson, F. Pfeiffer, O. Bunk, C. David, K. Jefimovs, and I. Johnson, Phys. Rev. Lett. 98, 034801 (2007).

[7] R. W. Gerchberg and W. O. Saxton, Optik (Stuttgart) 35, 237 (1972).

[8] J. R. Fienup, Appl. Opt. 21, 2758 (1982).

[9] H. A. Hauptman, Rep. Prog. Phys. 54, 1427 (1991).

[10] P. Coles, P. Dineen, J. Earl, and D. Wright, Mon. Not. R. Astron. Soc. 350, 989 (2004).

[11] L.-Y. Chiang and P. D. Naselsky, Mon. Not. R. Astron. Soc. 380, L71 (2007).

[12] L.-Y. Chiang, P. D. Naselsky, and P. Coles, Astrophys. J. 664, 8 (2007).
[13] A. H. Guth, Phys. Rev. D 23, 347 (1981).

[14] A. Albrecht and P. J. Steinhardt, Phys. Rev. Lett. 48, 1220 (1982).

[15] A. D. Linde, Phys. Lett. 108B, 389 (1982).

[16] P. A. R. Ade et al.. (Planck Collaboration), arXiv: 1303.5084.

[17] J. Theiler, S. Eubank, A. Longtin, B. Galdrikian, and J. D. Farmer, Physica (Amsterdam) 58D, 77 (1992).

[18] P. A. R. Ade et al. (Planck Collaboration), arXiv:1303.5083.

[19] C. Räth, A. J. Banday, G. Rossmanith, H. Modest, R. Sütterlin, K. M. Górski, J. Delabrouille, and G. E. Morfill, Mon. Not. R. Astron. Soc. 415, 2205 (2011).

[20] G. Rossmanith, H. Modest, C. Räth, A. J. Banday, K. M. Górski, and G. Morfill, Phys. Rev. D 86, 083005 (2012).

[21] H. I. Modest, C. Räth, A. J. Banday, G. Rossmanith, R. Sütterlin, S. Basak, J. Delabrouille, K. M. Górski, and G. E. Morfill, Mon. Not. R. Astron. Soc. 428, 551 (2013).

[22] T. R. Jaffe, A. J. Banday, H. K. Eriksen, K. M. Gorski, and F. K. Hansen, Astrophys. J. Lett. 629, L1 (2005).

[23] L. Cayón, A. J. Banday, T. Jaffe, H. K. Eriksen, F. K. Hansen, K. M. Gorski, and J. Jin, Mon. Not. R. Astron. Soc. 369, 598 (2006).

[24] J. D. McEwen, M. P. Hobson, A. N. Lasenby, and D. J. Mortlock, Mon. Not. R. Astron. Soc. 369, 1858 (2006).

[25] T. R. Jaffe, S. Hervik, A. J. Banday, and K. M. Górski, Astrophys. J. 644, 701 (2006).

[26] J. D. Barrow, R. Juszkiewicz, and D. H. Sonoda, Mon. Not. R. Astron. Soc. 213, 917 (1985).

[27] N. Wiener, Acta Math. 55, 117 (1930).

[28] A. I. Khinchin, Mathematical Foundations of Information Theory (Dover, New York, 1957). 
[29] C. L. Bennett, D. Larson, J. L. Weiland, N. Jarosik, G. Hinshaw, N. Odegard, K. M. Smith, R. S. Hill, B. Gold, M. Halpern, E. Komatsu, M. R. Nolta, L. Page, D. N. Spergel, E. Wollack, J. Dunkley, A. Kogut, M. Limon, S. S. Meyer, G. S. Tucker, and E. L. Wright, Astrophys. J. Suppl. Ser. 208, 20 (2013).

[30] T. R. Jaffe, A. J. Banday, H. K. Eriksen, K. M. Górski, and F. K. Hansen, Astron. Astrophys. 460, 393 (2006).

[31] J. D. McEwen, T. Josset, S. M. Feeney, H. V. Peiris, and A. N. Lasenby, Mon. Not. R. Astron. Soc. 436, 3680 (2013).

[32] P. A. R. Ade et al.. (Planck Collaboration), arXiv:1303.5086.

[33] http://www.sciops.esa.int/index.php?project=planck\&page= Planck_Legacy_Archive.

[34] http://lambda.gsfc.nasa.gov/.

[35] N. H. Kuiper, KNAW Proceedings Series A 63, 38 (1960).

[36] W. H. Press, S. A. Teukolsky, W. T. Vetterling, and B. P. Flannery, Numerical Recipes: The Art of Scientific Computing, 3rd ed. (Cambridge University Press, Cambridge, England, 2007).

[37] C. Räth and G. Morfill, J. Opt. Soc. Am. A 14, 3208 (1997).
[38] C. Räth, M. Gliozzi, I. E. Papadakis, and W. Brinkmann, Phys. Rev. Lett. 109, 144101 (2012).

[39] H. Minkowski, Math. Ann. 57, 447 (1903).

[40] K. R. Mecke, T. Buchert, and H. Wagner, Astron. Astrophys. 288, 697 (1994).

[41] S. Winitzki and A. Kosowsky, New Astron. 3, 75 (1998).

[42] J. Schmalzing and K. M. Górski, Mon. Not. R. Astron. Soc. 297, 355 (1998).

[43] See Supplemental Material at http://link.aps.org/ supplemental/10.1103/PhysRevD.89.123004 for the results of averaging over 768 coordinate systems.

[44] F. K. Hansen, A. J. Banday, K. M. Górski, H. K. Eriksen, and P. B. Lilje, Astrophys. J. 704, 1448 (2009).

[45] A. Ben-David, E. D. Kovetz, and N. Itzhaki, Astrophys. J. 748, 39 (2012).

[46] C. J. Copi, D. Huterer, D. J. Schwarz, and G. D. Starkman, arXiv:1311.4562.

[47] C. J. Copi, D. Huterer, D. J. Schwarz, and G. D. Starkman, arXiv:1310.3831.

[48] K. M. Górski, E. Hivon, A. J. Banday, B. D. Wandelt, F. K. Hansen, M. Reinecke, and M. Bartelmann, Astrophys. J. 622, 759 (2005). 